\title{
Lorentz covariant form of extended higher-spin equations
}

\author{
V.E. Didenko, ${ }^{a}$ N.G. Misuna ${ }^{a, b}$ and M.A. Vasiliev ${ }^{a}$ \\ ${ }^{a}$ I.E. Tamm Department of Theoretical Physics, Lebedev Physical Institute, \\ Leninsky prospect 53, 119991, Moscow, Russia \\ ${ }^{b}$ Moscow Institute of Physics and Technology, \\ Institutsky lane 9, 141700, Dolgoprudny, Moscow region, Russia \\ E-mail: didenko@lpi.ru, misuna@phystech.edu, vasiliev@lpi.ru
}

ABSTRACT: The extension of nonlinear higher-spin equations in $d=4$ proposed in [30] for the construction of invariant functional is shown to respect local Lorentz symmetry. The equations are rewritten in a manifestly Lorentz covariant form resulting from some Stueckelberg-like field transformation. We also show that the two field-independent central terms entering higher-spin equations which are not entirely fixed by the consistency alone get fixed unambiguously by the requirement of Lorentz symmetry. One of the important advantages of the proposed approach demonstrated in the paper is the remarkable simplification of the perturbative analysis.

KEYwords: Gauge Symmetry, Higher Spin Symmetry

ARXIV EPRINT: 1712.09272 


\section{Contents}

1 Introduction 1

2 Lorentz covariant HS equations $\quad 4$

3 Covariantization of extended higher-spin system $\quad 9$

3.1 Lorentz covariance in the twistor sector $\quad 9$

$\begin{array}{lll}3.2 & \text { Generalized deformed oscillator algebra } & 11\end{array}$

$\begin{array}{lll}3.3 & \text { Lorentz covariant equations } & 12\end{array}$

4 Covariant perturbation theory 13

$\begin{array}{lll}4.1 \text { Adjoint case } & 14\end{array}$

$\begin{array}{ll}4.2 \text { Twisted-adjoint case } & 17\end{array}$

$\begin{array}{llr}5 & \text { Conclusion } & 18\end{array}$

\section{Introduction}

Nonlinear higher-spin (HS) equations were presented [1,2] in the unfolded form of covariant first-order differential equations. Generalizing Maurer-Cartan equations, unfolded equations [3, 4] (for reviews see e.g. [5, 6]) manifestly control gauge symmetries and diffeomorphism invariance. This is achieved due to appearance of infinite towers of auxiliary fields packed into HS master fields, that describe all on-shell derivatives of HS fields. Schematically any unfolded system has the form

$$
\mathrm{d} W(x)=F(W(x))
$$

where $W(x)$ is some set of differential form fields and d is the space-time De Rham differential. (All products are wedge products. The wedge symbol is implicit.) Unfolded equations (1.1) are consistent if no other than (1.1) conditions result from the integrability requirement $\mathrm{d}^{2}=0$.

Among different approaches to HS dynamics, one of the most natural is the metriclike one pioneered by Fronsdal [7]. However, even though it aims at generalization of the Einstein gravity in terms of habitual tensor fields, the progress in the construction of interactions is rather limited beyond the cubic (Lagrangian) order (see e.g. [8]-[12]) due to the technical challenge and lack of geometric intuition within this formalism. At least to lower orders, however, the HS AdS/CFT holography allows reconstructing local HS interactions [13]. The situation with the light-cone approach is analogous [14]-[23] with the notable exception for the paper by Metsaev [24] who was able to deduce nontrivial restrictions on the cubic vertex from the analysis of quartic interactions. 
The frame-like approach initiated in $[25,26]$ is a HS generalization of the Cartan formulation of gravity. It naturally generalizes the spin-two curvature two-forms to higher spins the same time bringing in a new piece of information such as the notion of HS algebra and tightly related ingredient, spectrum of auxiliary fields. In the frame-like approach both covariant HS cubic couplings [27] and fully nonlinear HS equations were found [1, 2]. Its relation to the metric-like language is due to the local Lorentz symmetry which enables one rewriting field components from the frame-like setting to the metric-like and vice versa. (Recall that in Cartan's gravity the equivalence principle rests on both diffeomorphism invariance and local Lorentz symmetry.)

Retrospectively, in formulating nonlinear HS equations the guiding rules were the consistency of (1.1) and the correct free limit about AdS background which is the exact vacuum solution. The former automatically guarantees gauge invariance. Diffeomorphism invariance was by construction. Local Lorentz symmetry was also inbuilt by considering the problem in terms of multispinors as finite-dimensional Lorentz modules. All these properties were inherited by the nonlinear HS equations of $[1,2]$. Somewhat later it was realized [5] that the form of equations of [2] is to large extent fixed by the local Lorentz covariance principle that can be easily lost by an otherwise consistent deformation of the equations of [2].

Local Lorentz covariance implies that the equations should be of the form

$$
D^{L} W=F^{L}(W)
$$

where $D^{L}=\mathrm{d}+\omega^{L}$ is the Lorentz covariant derivative acting in the standard way on any Lorentz tensor (multispinor) with the convention that $D^{L} \omega^{L}$ is identified with the Lorentz curvature $R^{L}=\mathrm{d} \omega^{L}+\omega^{L} \wedge \omega^{L}$. Then local Lorentz symmetry demands that the Lorentz connection $\omega^{L}$ does not enter the r.h.s. of (1.2). Practically, it is more convenient to work with (1.1) than with (1.2) since $\left(D^{L}\right)^{2} \neq 0$ while $\mathrm{d}^{2}=0$. It is therefore necessary to check if the unfolded equations (1.1) admit a field redefinition to (1.2) to respect the local Lorentz covariance. That such a field redefinition is indeed available for HS systems in all orders in interactions has been shown in [5, 28] (see also [29]).

Recently, an extension of $4 d$ HS equations that contains HS invariant functionals has been proposed in [30]. One of such functionals conjectured to give an on-shell action is anticipated to play a crucial role for the purposes of HS AdS/CFT industry (see [31]-[41] for the incomplete list of references). In view of absence of conventional fully nonlinear HS action principle (see however [42]) the possibility of calculating the on-shell action paves a way to testing HS AdS/CFT proposal explicitly at least at tree level. (A further extension accounting for quantum corrections was also discussed in [30].) The extension of [30] is designed to include higher-degree differential forms in addition to the zero- and one-forms of the original HS theory. These higher forms carry no local physical degrees of freedom being expressed in terms of physical fields on mass shell. The space-time four-form valued in the center of the HS algebra corresponds to the density of the aforementioned on-shell action.

To calculate the HS invariant density and compare it with the correlation functions of the dual theory in the boundary limit is a challenging technical problem even to the lowest orders. The main complication is due to the involved nature of HS perturbation theory despite considerable progress made in [43]. A related difficulty is the fact that the 
extended HS equations have been written down in form (1.1) rather than in (1.2) leading to non-covariant and highly involved expressions for the invariant functional requiring further field redefinition for the covariantization.

The existence of Lorentz covariant frame for the extended HS equations was neither presented nor proven in the literature. In this paper we fill in this gap providing the explicit Lorentz covariant extended HS equations in four dimensions. One of the main difficulties in obtaining these equations is the fact that the Lorentz covariant differential $D^{L}$ does not admit star-product realization in its Lorentz connection part. This makes it hard finding field redefinition that lead to the covariant form of equations. Instead of looking for such a field redefinition, our strategy is to start from the part of the equations for space-time zero-forms which can be straightforwardly covariantized. Consistency of these equations imposes certain restrictions on the form of the rest of the equations still not constraining them entirely. At this stage the analysis requires a bit of a guess work. An important observation is that the very requirement of local Lorentz symmetry restricts the form of field-independent central terms in the equations that determine the structure of interactions, which otherwise are fixed by rather indirect functional class arguments and not by mere consistency of HS equations. The availability of a Lorentz frame largely relies on the existence of field-dependent Lorentz generators constructed out of purely twistor sector of the extended HS equations thanks to their specific form that admits interpretation in terms of a deformed oscillator algebra generalizing the deformed oscillator realization of the Lorentz algebra underlying usual HS equations of [2] (see also [5] and references therein).

Lorentz covariant equations proposed in this paper contain an additional Lorentz connection on the top of the tower of HS gauge fields. Their integrability results in an overdetermined set of constraints which are however fulfilled as a consequence of spinor Fierz (Schouten) identities. To see that the proposed equations are dynamically equivalent to those of [30] we observe additional Stueckelberg symmetry generalizing that found in [44] for the usual bosonic HS equations, which allows one to set an additional Lorentz connection to zero thus reducing equations to their ordinary unfolded frame yet providing explicit field redefinition relating the two frames. Naively, Stueckelberg nature of the spin-two Lorentz connection in HS master field implies that it is gauge equivalent to zero. Once however we insist on the absence of the Lorentz connection type contribution to the spin two sector within HS master field, then the additional Lorentz connection gets perturbatively determined up to true HS gauge symmetry transformations.

From technical standpoint the advantage of the proposed covariant equations as compared to those in [30] is due to a remarkable simplification of perturbative series operators they deliver along the lines of [43]. To show this we reconsider perturbative expansion about proper HS vacuum solution within the Lorentz covariant approach and rederive operators which determine perturbative analysis of the equations of motion. Our result reproduces formulas of [43] with the difference that they no longer contain Lorentz connection, leading to dramatic simplification especially for the twisted-adjoint case.

The paper is organized as follows. In section 2 we recall HS equations in four dimensions and explain a way of making them explicitly Lorentz covariant suitable for the HS 
system containing the invariant functional. Then, in section 3 we address a problem of covariantization of the extended HS equations. To do that we discuss a generalization of the deformed oscillator algebra in section 3.2. A manifestly covariant form of HS equations is given in section 3.3. Finally, in section 4 we elaborate a covariant perturbation theory for the obtained equations. Brief conclusions are in section 5.

\section{Lorentz covariant HS equations}

Let us start by reviewing the HS equations in four dimensions of [2] (see also [5]) and their Lorentz covariantization. The equations have the following standard form

$$
\begin{aligned}
& \mathrm{d} \mathcal{W}+\mathcal{W} * \mathcal{W}=i \theta^{A} \theta_{A}+i \eta B * \gamma+i \bar{\eta} B * \bar{\gamma} \\
& \mathrm{d} B+[\mathcal{W}, B]_{*}=0,
\end{aligned}
$$

where $\mathcal{W}(Z, Y ; K \mid x)$ is the 1 -form in the double graded space spanned by anticommuting $\mathrm{d} x^{\underline{m}}$ and auxiliary $\theta^{A}$ differentials. $\mathcal{W}$ contains HS potentials with all their descendants in space-time subsector $W$ and the compensator-like field $S$ in the $\theta$-subsector,

$$
\begin{aligned}
\mathcal{W}(Z, Y ; K \mid x) & =W_{\underline{m}}(Z, Y ; K \mid x) \mathrm{d} x^{\underline{\underline{m}}}+S_{A}(Z, Y ; K \mid x) \theta^{A}, \\
\left\{\mathrm{~d} x^{\underline{m}}, \mathrm{~d} x^{\underline{n}}\right\} & =\left\{\mathrm{d} x^{\underline{m}}, \theta^{A}\right\}=\left\{\theta^{A}, \theta^{B}\right\}=0 .
\end{aligned}
$$

Space-time indices $\underline{m}, \underline{n}$ as well as Majorana spinor fiber indices $A, B$ range four values. $B(Z, Y ; K \mid x)$ is a 0 -form master field, containing lower spin matter fields and HS curvatures.

Apart from $x$-dependence, $\mathcal{W}$ and $B$ depend on a number of generating variables. Commuting twistor-like variables $Y_{A}=\left(y_{\alpha}, \bar{y}_{\dot{\alpha}}\right)$ and $Z_{A}=\left(z_{\alpha}, \bar{z}_{\dot{\alpha}}\right)$ are designed to pack up HS fields and auxiliary fields, where spinor indices $\alpha, \beta, \ldots$ range two values. The associative star-product operation acts on functions $f(Z, Y)$

$$
(f * g)(Z, Y)=\frac{1}{(2 \pi)^{4}} \int d U d V f(Z+U, Y+U) g(Z-V, Y+V) e^{i U_{A} V^{A}}
$$

where $U_{A} V^{A}:=U_{A} V_{B} \epsilon^{A B}$ with some $s p(4)$-invariant symplectic form $\epsilon_{A B}=-\epsilon_{B A}$. Indices are raised and lowered with the aid of $\epsilon_{A B}$ as follows, $X^{A}=\epsilon^{A B} X_{B}$ and $X_{A}=\epsilon_{B A} X^{B}$. The star product can be seen to induce the following commutation relations

$$
\left[Y_{A}, Y_{B}\right]_{*}=-\left[Z_{A}, Z_{B}\right]_{*}=2 i \epsilon_{A B}, \quad\left[Y_{A}, Z_{B}\right]_{*}=0 .
$$

It admits the inner Klein operators

$$
\varkappa=e^{i z_{\alpha} y^{\alpha}}, \quad \bar{\varkappa}=e^{i \bar{z}_{\dot{\alpha}} \bar{y}^{\dot{\alpha}}} .
$$

Their characteristic properties are

$$
\left\{\varkappa, y_{\alpha}\right\}_{*}=\left\{\varkappa, z_{\alpha}\right\}_{*}=0, \quad \varkappa * \varkappa=1,
$$

analogously in the antiholomorphic sector for $\bar{\varkappa}$. 
To distinguish between the adjoint representation for HS potentials and the twistedadjoint for HS curvatures as well as to have a room for topological degrees of freedom in HS system one introduces Clifford variables $K=(k, \bar{k})$ which are called the outer Klein operators. $k$ anticommutes with the holomorphic variables $y_{\alpha}, z_{\alpha}, \theta_{\alpha}$ and commutes with every antiholomorhic one. Note that being similar to star-product realized $\varkappa, k$ nevertheless admits no algebraic realization due to its anticommutativity with $\theta^{\alpha}$. Analogously, $\bar{k}$ anticommutes with antiholomorhic variables and commutes with all of the rest. In addition,

$$
k^{2}=\bar{k}^{2}=1, \quad[k, \bar{k}]=0 .
$$

The latter relation makes the dependence on Klein operators at most bilinear

$$
\mathcal{W}=\sum_{i, j=0,1} \mathcal{W}_{i, j} k^{i} \bar{k}^{j}, \quad B=\sum_{i, j=0,1} B_{i, j} k^{i} \bar{k}^{j}
$$

Physical fields are encoded in $\mathcal{W}(-k,-\bar{k})=\mathcal{W}(k, \bar{k})$ and $B(-k,-\bar{k})=-B(k, \bar{k})$. The rest decompose into infinite tower of fields, each carrying at most a finite number of dynamical degrees of freedom, being therefore topological.

There are two central elements that enter the r.h.s. of (2.1). One is $\theta^{A} \theta_{A}$ which obviously commutes with any variable. Another one is

$$
\gamma=2 k \varkappa \delta^{2}(\theta), \quad \delta^{2}(\theta):=\frac{1}{2} \theta^{\alpha} \theta_{\alpha},
$$

which commutes with everything including $\theta^{\alpha}$ thanks to the Grassmann $\delta$-function, $\theta^{\alpha} \delta^{2}(\theta)=0$. Analogously defined is $\bar{\gamma}$. Finally, complex phase $\eta=e^{i \phi}$ is the only free parameter of HS theory in four dimensions with the r.h.s. of (2.1) linear in $B$. For parity preserving cases when $\phi=0$ the theory is known as the A-model and $\phi=\pi / 2$ corresponds to the B-model [32].

In terms of $W$ and $S$ components of $\mathcal{W}(2.1),(2.2)$ can be rewritten as follows

$$
\begin{aligned}
\mathrm{d} W+W * W & =0, \\
\mathrm{~d} S+[W, S]_{*} & =0, \\
\mathrm{~d} B+[W, B]_{*} & =0, \\
S * S & =-i \theta_{\alpha} \wedge \theta^{\alpha}(1+\eta B * k \varkappa)-i \bar{\theta}_{\dot{\alpha}} \wedge \bar{\theta}^{\dot{\alpha}}(1+\bar{\eta} B * \bar{k} \bar{\varkappa}), \\
{[S, B]_{*} } & =0 .
\end{aligned}
$$

To proceed to Lorentz covariance let us first recall that Lorentz covariant derivative of a Lorentz multispinor $\phi_{\alpha_{1} \ldots \alpha_{n}, \dot{\alpha}_{1} \ldots \dot{\alpha}_{m}}$ is

$$
D^{L} \phi_{\alpha(n), \dot{\alpha}(m)}=\mathrm{d} \phi_{\alpha(n), \dot{\alpha}(m)}-n \omega_{\alpha}^{\beta} \phi_{\beta \alpha(n-1), \dot{\alpha}(m)}-m \bar{\omega}_{\dot{\alpha}}^{\dot{\beta}} \phi_{\alpha(n), \dot{\beta} \dot{\alpha}(m-1)},
$$

where $\omega_{\alpha \beta}$ and $\bar{\omega}_{\dot{\alpha} \dot{\beta}}$ are holomorphic and antiholomorphic parts of the Lorentz connection $\omega^{A B}=\left(\omega_{\alpha \beta}, \bar{\omega}_{\dot{\alpha} \dot{\beta}}\right)$ which generates Lorentz curvature 2-form via

$$
\begin{aligned}
\left(D^{L}\right)^{2} \phi_{\alpha(n), \dot{\alpha}(m)} & =-n R_{\alpha}{ }^{\beta} \phi_{\beta \alpha(n-1), \dot{\alpha}(m)}-m \bar{R}_{\dot{\alpha}}^{\dot{\beta}} \phi_{\alpha(n), \dot{\beta} \dot{\alpha}(m-1)}, \\
R_{\alpha \beta} & =\mathrm{d} \omega_{\alpha \beta}-\omega_{\alpha}^{\gamma} \wedge \omega_{\gamma \beta}, \\
D^{L} R_{\alpha \beta} & =D^{L} \bar{R}_{\dot{\alpha} \dot{\beta}}=0 .
\end{aligned}
$$


In HS equations (2.12)-(2.16) all spin-tensors are packed into generating functions $W$, $S$ and $B$ with the aid of the variables $Y^{A}, Z^{A}$ and also $\theta^{A}$ in case of $S$-field. In these terms, (2.17) can be rewritten as

$$
D^{L} f(Z, Y ; \theta)=\left(\mathrm{d}+\omega^{A B}\left(Z_{A} \frac{\partial}{\partial Z^{B}}+Y_{A} \frac{\partial}{\partial Y^{B}}+\theta_{A} \frac{\partial}{\partial \theta^{B}}\right)\right) f(Z, Y ; \theta) .
$$

Note that the differential with respect to $Y$ and $Z$ operators in (2.21) can be realized in terms of star product

$$
\begin{aligned}
\omega^{A B}\left(Z_{A} \frac{\partial}{\partial Z^{B}}+Y_{A} \frac{\partial}{\partial Y^{B}}\right) & =\omega^{A B}\left[L_{A B}, f\right]_{*}, \\
L_{A B} & =-\frac{i}{4}\left(Y_{A} Y_{B}-Z_{A} Z_{B}\right) .
\end{aligned}
$$

Clearly, $L_{\alpha \beta}$ and $\bar{L}_{\dot{\alpha} \dot{\beta}}$ form $s l_{2}(\mathbb{C})$ Lorentz subalgebra. It follows then that if it were not for $\theta$-dependence in $S$ field the local Lorentz symmetry on HS equations could be restored by a simple field redefinition $W \rightarrow W+\omega^{A B} L_{A B}$. Indeed, in this case the de-Rham differential $\mathrm{d}$ in (2.13) and (2.14) turns into the Lorentz differential $D^{L}$ by the $\omega^{A B} L_{A B}$ shift. However this shift does not act properly on $S$ field in (2.13) which being $\theta$-dependent carries extra Lorentz indices $S=S_{\alpha} \theta^{\alpha}+\bar{S}_{\dot{\alpha}} \bar{\theta}^{\dot{\alpha}}$ not rotated by $L_{A B}$. This is where the last term in (2.21) becomes important. Since $\theta_{A} \frac{\partial}{\partial \theta^{B}}$ cannot be realized via star-product there must be a special reason for the local Lorentz symmetry to take place. ${ }^{1}$ As shown in $[5,28]$ Lorentz covariance follows from the property of the deformed oscillator algebra as we now recall.

For the sake of presentation simplicity, consider for a moment bosonic truncation with no topological degrees of freedom in (2.12)-(2.16). General case is fully captured by this example. We are interested in the $\theta$-sector, i.e. the sector of space-time 0 -forms to be referred to as the twistor sector. Corresponding equations are

$$
\begin{aligned}
{\left[S_{\alpha}, S_{\beta}\right]_{*} } & =-2 i \epsilon_{\alpha \beta}(1+\eta B * \varkappa), \\
\left\{S_{\alpha}, B * \varkappa\right\}_{*} & =0,
\end{aligned}
$$

(similarly in the dotted sector) where fields $S$ and $B$ are now $k$ and $\bar{k}$ independent. Equations (2.24)-(2.25) have the form of deformed oscillator algebra $[45,46]$ where it was observed that it respects $s p(2)$ symmetry. Namely, the generators

$$
M_{\alpha \beta}=\frac{i}{8}\left\{S_{\alpha}, S_{\beta}\right\}_{*}
$$

form $s p(2)$ for any $B$,

$$
\left[M_{\alpha \alpha}, M_{\beta \beta}\right]_{*}=2 \epsilon_{\alpha \beta} M_{\alpha \beta} .
$$

Together with the generators in the antiholomorphic sector it restores the full Lorentz algebra $s l_{2}(\mathbb{C})$. Particularly, by $(2.24),(2.26)$ these generators Lorentz rotate the field $S$ itself

$$
\left[M_{\alpha \alpha}, S_{\beta}\right]_{*}=\epsilon_{\alpha \beta} S_{\alpha} .
$$

\footnotetext{
${ }^{1} \mathrm{~A}$ naive way out is to extend star product spanned by bosonic $Y$ and $Z$ variables by adding Clifford elements $\theta$. Such an extension while making scheme explicitly Lorentz covariant appears to change the content of the equations.
} 
Since (2.28) mimics the action of the $\theta$-term in (2.21) this suggests that the field-dependent generators $M_{\alpha \beta}$ and $\bar{M}_{\dot{\alpha} \dot{\beta}}$ can underly the field redefinition that restores local Lorentz symmetry. Indeed, at the nonlinear level the Lorentz algebra action consists of two parts (2.23) and (2.28) suggesting the following field redefinition $W \rightarrow W+\omega^{A B}\left(L_{A B}+M_{A B}\right)$. That this is indeed the case can be checked directly (see $[5,29]$ ). We however take somewhat different way which is most useful in the more complicated extended HS system.

Let us assume that there exists a field redefinition that makes local Lorentz symmetry manifest. Since such redefinition contains Lorentz connection it cannot affect space-time 0forms $S$ and $B$. This implies that our bosonic truncated equations (2.24) and (2.25) remain undeformed. The only field that should be redefined is the space-time 1-form $W$. The effect of the field redefinition $W \rightarrow W^{\prime}=W+\omega^{A B}(\ldots)$ on the zero-curvature conditions

$$
\begin{aligned}
\mathrm{d} S_{\alpha}+\left[W, S_{\alpha}\right]_{*} & =0, \\
\mathrm{~d} B+W * B-B * \pi(W) & =0,
\end{aligned}
$$

where $\pi(y, \bar{y}, z, \bar{z})=(-y, \bar{y},-z, \bar{z})$ will be their covariantization of the form

$$
\begin{aligned}
D^{L} S_{\alpha}+\left[W^{\prime}, S_{\alpha}\right]_{*} & =0, \\
D^{L} B+W^{\prime} * B-B * \pi\left(W^{\prime}\right) & =0
\end{aligned}
$$

with $W^{\prime}$ being free from the Lorentz connection. Consistency (2.18)

$$
\left(D^{L}\right)^{2} S_{\alpha}=R^{\beta \gamma}\left[L_{\beta \gamma}, S_{\alpha}\right]_{*}-R_{\alpha}^{\beta} S_{\beta}
$$

applied to (2.31) implies using (2.26) that

$$
\left[D^{L} W^{\prime}+W^{\prime} * W^{\prime}+R^{\beta \gamma}\left(L_{\beta \gamma}-\frac{i}{4} S_{\beta} * S_{\gamma}\right), S_{\alpha}\right]_{*}=0
$$

suggesting eventually the following Lorentz covariant system

$$
\begin{aligned}
& D^{L} W^{\prime}+W^{\prime} * W^{\prime}+R^{A B}\left(L_{A B}-\frac{i}{4} S_{A} * S_{B}\right)=0, \\
& R^{A B}:=\mathrm{d} \omega^{A B}-\omega^{A C} \omega_{C}{ }^{B}, \\
& D^{L} S_{A}+\left[W^{\prime}, S_{A}\right]_{*}=0, \\
& D^{L} B+W^{\prime} * B-B * \pi\left(W^{\prime}\right)=0, \\
& {\left[S_{\alpha}, S_{\beta}\right]_{*}=-2 i \epsilon_{\alpha \beta}(1+\eta B * \varkappa), \quad\left[\bar{S}_{\dot{\alpha}}, \bar{S}_{\dot{\beta}}\right]_{*}=-2 i \epsilon_{\dot{\alpha} \dot{\beta}}(1+\bar{\eta} B * \bar{\varkappa}),} \\
& \left\{S_{\alpha}, B * \varkappa\right\}_{*}=0, \quad\left\{\bar{S}_{\dot{\alpha}}, B * \bar{\varkappa}\right\}_{*}=0 .
\end{aligned}
$$

The obtained equations are consistent and exhibit manifest local Lorentz symmetry. In this form they were written down in [29]. By the field redefinition

$$
W^{\prime}=W-\omega^{A B}\left(L_{A B}-\frac{i}{4} S_{A} * S_{B}\right)
$$

Eqs. (2.35)-(2.40) is reduced down to the bosonic version of (2.12)-(2.16). 
Before we pursue covariantization of general case (2.1), (2.2) let us make few comments. First, we see that twistor sector of HS equations plays crucial role for local Lorentz symmetry. Namely, the possibility to write down covariant equations through a field redefinition relies on the existence of field dependent Lorentz generators built from the on-shell twistor fields (see (2.28)). For example, while one can relax (2.1) by dropping off $\theta^{A} \theta_{A}$ term still keeping equations consistent ${ }^{2}$ there would be no local Lorentz symmetry as we will see. We will keep this strategy of examining twistor sector in our analysis of more complicated HS extended equations.

Second, the obtained equations (2.35)-(2.40) do not determine Lorentz connection $\omega_{A B}$ by itself. As shown in [44] where the Lorentz covariant equations were used, this system possesses Stückelberg symmetry that allows one to gauge away the connection. The connection nonetheless can be perturbatively fixed up to HS gauge transformations once the field $W^{\prime}$ is demanded to be free of connection-type components. This implies that the physical Lorentz connection given by $\omega_{A B}$ is unique. To be more specific, normally one associates physical fields with cohomologies represented by $Z$-independent functions (see e.g., $[5,29]$ ). Therefore we need no (anti)holomorphic bilinears to reside in $W^{\prime}$ which if present would correspond to yet another connection-type fields. Hence, we set

$$
\left.\frac{\partial^{2}}{\partial y^{\alpha} \partial y^{\beta}} W^{\prime}\right|_{Z, Y=0}=\left.\frac{\partial^{2}}{\partial \bar{y}^{\dot{\alpha}} \partial \bar{y}^{\dot{\beta}}} W^{\prime}\right|_{Z, Y=0}=0 .
$$

Now let us consider generic case of HS equations that contain double set of physical fields along with the topological ones in accordance with (2.1)-(2.2). To make them Lorentz covariant we use the following trick. We note that the $S$-term in parentheses of (2.35) can be formally represented as $\frac{\partial}{\partial \theta^{A}} \mathcal{W}$. This operator however does not respect the chain rule on a product of fields due to their dependence on outer Klein operators $k$ and $\bar{k}$. To fix that we introduce an additional operator $\rho$ that anticommutes with $k$ and commutes with the rest (similar operator appears in $3 d$ model of [47]) and analogously we introduce $\bar{\rho}$ that anticommutes with $\bar{k}$

$$
\rho k+k \rho=0, \quad \rho \theta-\theta \rho=0, \quad \rho^{2}=1 .
$$

These properly account for sign flip of the outer Klein operators $k$ and $\bar{k}$ in their master field dependence allowing to define derivations

$$
\partial_{\alpha}:=\rho \frac{\partial}{\partial \theta^{\alpha}}, \quad \partial_{\dot{\alpha}}:=\bar{\rho} \frac{\partial}{\partial \bar{\theta}^{\dot{\alpha}}}
$$

respecting the chain rule

$$
\partial_{A}\left(F^{(p)} * G^{(q)}\right)=\partial_{A} F^{(p)} * G^{(q)}+(-)^{p} F^{(p)} * \partial_{A} G^{(q)}
$$

and allowing one to extend the bosonic case to the following general Lorentz covariant HS equations

$$
\begin{aligned}
D^{L} \mathcal{W}+\mathcal{W} * \mathcal{W}+R^{A B}\left(L_{A B}-\frac{i}{4} \partial_{A} \mathcal{W} * \partial_{B} \mathcal{W}\right) & =i \theta^{A} \theta_{A}+i \eta B * \gamma+i \bar{\eta} B * \bar{\gamma} \\
D^{L} B+[\mathcal{W}, B] & =0
\end{aligned}
$$

\footnotetext{
${ }^{2}$ This example is physics wise unappropriate due to its failure of reproducing free Fronsdal equations.
} 
Note that the dependence on $\rho$ and $\bar{\rho}$ drops out from (2.46) upon generating certain sign factors. The consistency of (2.46), (2.47) is easy to check using

$$
\begin{aligned}
\left(D^{L}\right)^{2} f(Z, Y ; \theta) & =R^{A B}\left[L_{A B}, f\right]+R^{A B} \theta_{A} \frac{\partial}{\partial \theta^{B}} f, \\
D^{L}\left(R^{A B} L_{A B}\right) & =0 .
\end{aligned}
$$

For $\omega_{A B}=0$ one gets back to (2.1), (2.2). For $\omega_{A B} \neq 0$ following [44] we observe an extra Stückelberg symmetry

$$
\begin{aligned}
\delta_{\xi} \omega_{A B} & =\xi_{A B}, \quad \delta_{\xi} B=0 \\
\delta_{\xi} \mathcal{W} & =-\xi^{A B}\left(L_{A B}-\frac{i}{4} \partial_{A} \mathcal{W} * \partial_{B} \mathcal{W}\right) .
\end{aligned}
$$

It is interesting to note, that by simple rescaling of $\theta$-variables along with master fields one can modify the first term on the r.h.s. of (2.1) to be $i \nu \theta^{A} \theta_{A}$, where $\nu$ is an arbitrary real number. This rescaling correspondingly modifies covariant equation (2.46)

$$
D^{L} \mathcal{W}+\mathcal{W} * \mathcal{W}+R^{A B}\left(L_{A B}-\frac{i}{4 \nu} \partial_{A} \mathcal{W} * \partial_{B} \mathcal{W}\right)=i \nu \theta^{A} \theta_{A}+i \eta B * \gamma+i \bar{\eta} B * \bar{\gamma}
$$

which makes the limit $\nu \rightarrow 0$ meaningless unlike the same limit for the noncovariant system. Therefore, the very requirement of Lorentz symmetry rules out some formally consistent systems. This particular limit can be ruled out by comparing the free equations it provides to the proper free HS ones though. In other words (2.46) written down in the non-covariant frame $R^{A B}=0$ does not reproduce proper free HS equations upon linearization about AdS background for $\nu=0$.

\section{Covariantization of extended higher-spin system}

\subsection{Lorentz covariance in the twistor sector}

An extended version of HS equations in four dimensions was proposed in [30]. Being physically equivalent to the original HS equations it contains higher differential forms which are expressed on-shell via dynamical HS fields. This extension is aimed at generating a gauge-invariant functional represented as a space-time integral over a four-form and is organized as follows.

The one-form $\mathcal{W}$ in $(\mathrm{d} x \oplus \theta)$-differentials is extended to three-forms $\mathrm{d} x^{n} \wedge \theta^{3-n}, n=$ $0, \ldots, 3$. Analogously, the zero-form $B(2.2)$ gets enhanced to all two-forms $\mathrm{d} x^{n} \wedge \theta^{2-n}, n=$ $0, \ldots, 2$. This way one introduces odd forms $\mathcal{W}$ and even forms $\mathcal{B}$. In different context a similar extension was also given in [42]. To let higher forms be nontrivially expressed in terms of dynamical HS fields of the original system, equations (2.1), (2.2) have to be properly modified since otherwise the higher forms would receive no non-zero source. The nontrivial HS dynamics in the original system rests on the sources bilinear in $\theta$ on the r.h.s. of (2.1) for the twistor field $S$. Now one has to introduce a source for a new twistor field resided in $\mathcal{W}$ which is a $\theta^{3}$-form. Compatibility of the modified equations demands 
any such source be central. Among few of such elements $g \gamma * \bar{\gamma}$, where $g$ is a constant, was argued in [30] to be the proper one. Eventually, the proposed equations are

$$
\begin{aligned}
\mathrm{d} \mathcal{W}+\mathcal{W} * \mathcal{W} & =i \theta^{A} \theta_{A}+i \eta \mathcal{B} * \gamma+i \bar{\eta} \mathcal{B} * \bar{\gamma}+i g \gamma * \bar{\gamma}+\mathcal{L} \\
\mathrm{d} \mathcal{B}+[\mathcal{W}, \mathcal{B}]_{*} & =0
\end{aligned}
$$

Here $\mathcal{L}$ is $Y, Z, k, \bar{k}$ and $\theta$-independent pure space-time four-form to be associated with the invariant functional density. ${ }^{3}$ Equation (3.1) may be deformed to contain $F_{*}(\mathcal{B})$ on the r.h.s. instead of $\mathcal{B}$ and $G(\mathcal{B})$ instead of $g$. We took $F=\eta \mathcal{B}, G(\mathcal{B})=g$ for simplicity. The system is invariant under the following gauge transformation

$$
\begin{aligned}
\delta \mathcal{W} & =\mathrm{d} \epsilon+[\mathcal{W}, \epsilon]_{*}+i \eta \xi * \gamma+i \bar{\eta} \xi * \bar{\gamma} \\
\delta \mathcal{B} & =\mathrm{d} \xi+\{\mathcal{W}, \xi\}_{*}+[\mathcal{B}, \epsilon]_{*} .
\end{aligned}
$$

Our goal is to prove that (3.1), (3.2) respects Lorentz symmetry, rewriting it in a manifestly Lorentz covariant form. A major asset on this way is the existence of Lorentz generators in the twistor sector of (3.1), (3.2) as we now show.

Let us introduce components for the twistor fields as follows

$$
\begin{gathered}
\left.\mathcal{W}\right|_{\mathrm{d} x=0}=s_{A} \theta^{A}+2 t_{\alpha} \theta^{\alpha} \delta^{2}(\bar{\theta})+2 \bar{t}_{\dot{\alpha}} \bar{\theta}^{\dot{\alpha}} \delta^{2}(\theta), \\
\left.\mathcal{B}\right|_{\mathrm{d} x=0}=B+2 b \delta^{2}(\theta)+2 \bar{b} \delta^{2}(\bar{\theta})+b_{\alpha \dot{\alpha}} \theta^{\alpha} \bar{\theta}^{\dot{\alpha}} .
\end{gathered}
$$

Assuming for brevity the bosonic truncation with $k \bar{k}=1$ from the $\theta$-sector of (3.1), (3.2) one extracts the following algebraic constraints

$$
\begin{aligned}
& {\left[s_{\alpha}, s_{\beta}\right]_{*}=-2 i \epsilon_{\alpha \beta}(1+\eta B * \varkappa), \quad\left[s_{\alpha}, \bar{s}_{\dot{\alpha}}\right]_{*}=0,} \\
& {\left[t_{\alpha}, s^{\alpha}\right]_{*}+\left[\bar{t}_{\dot{\alpha}}, \bar{s}^{\dot{\alpha}}\right]_{*}=-2 i(\eta \bar{b} * \varkappa+\bar{\eta} b * \bar{\varkappa}+g \varkappa * \bar{\varkappa}),} \\
& s_{\alpha} * B+B * \pi\left(s_{\alpha}\right)=0, \\
& s_{\alpha} * \bar{b}-\bar{b} * \bar{\pi}\left(s_{\alpha}\right)+t_{\alpha} * B+B * \pi\left(t_{\alpha}\right)=0, \\
& \frac{1}{2}\left(s_{\alpha} * b_{\dot{\alpha}}^{\alpha}+b_{\dot{\alpha}}^{\alpha} * \pi\left(s_{\alpha}\right)\right)+\bar{t}_{\dot{\alpha}} * B-B * \pi\left(\bar{t}_{\dot{\alpha}}\right)=0 .
\end{aligned}
$$

An important observation is that equations (3.7)-(3.11) admit Lorentz symmetry which can be realized as gauge transformation (3.3). This can be seen most easily for $\mathcal{B}=0$. Indeed from the gauge transformations

$$
\begin{aligned}
\delta_{\Lambda} s_{\alpha} & =\left[s_{\alpha}, \epsilon\right]_{*}, \\
\delta_{\Lambda} t_{\alpha} & =\left[t_{\alpha}, \epsilon\right]_{*}+\left[\phi, s_{\alpha}\right]+\left[\psi_{\alpha}{ }^{\dot{\beta}}, \bar{s}_{\dot{\beta}}\right], \\
\delta_{\Lambda} \bar{t}_{\dot{\alpha}} & =\left[\bar{t}_{\dot{\alpha}}, \epsilon\right]_{*}+\left[\bar{\phi}, \bar{s}_{\dot{\alpha}}\right]-\left[\bar{\psi}^{\beta}{ }_{\dot{\alpha}}, s_{\beta}\right],
\end{aligned}
$$

\footnotetext{
${ }^{3}$ Equivalently, one could introduce no $\mathcal{L}$ on the r.h.s. of (3.1) instead associating the four-form $(\mathcal{W} *$ $\mathcal{W})\left.\right|_{Z, Y, \theta=0}$ with the invariant functional density.
} 
where $\epsilon, \phi$ and $\psi_{\alpha \dot{\alpha}}$ are some gauge parameters, it follows that $\delta_{\Lambda} s_{\alpha}=\Lambda_{\alpha}{ }^{\beta} s_{\beta}$ and $\delta_{\Lambda} t_{\alpha}=$ $\Lambda_{\alpha}{ }^{\beta} t_{\beta}$ provided that

$$
\epsilon=-\frac{i}{4} \Lambda^{\alpha \beta} s_{\alpha} * s_{\beta}, \quad \phi=\frac{i}{4} \Lambda^{\alpha \beta}\left\{s_{\alpha}, t_{\beta}\right\}_{*}, \quad \psi_{\alpha \dot{\alpha}}=-\frac{i}{4} \Lambda_{\alpha}^{\beta}\left\{s_{\beta}, \bar{t}_{\dot{\alpha}}\right\}_{*} .
$$

This fact suggests that the whole system (3.1), (3.2) should be Lorentz covariant upon an appropriate field redefinition.

\subsection{Generalized deformed oscillator algebra}

Analogously to the original construction (2.24), (2.25) that underlies nonlinear HS equations, eqs. (3.7)-(3.11) give rise to a generalization of the deformed oscillator algebra.

Standard oscillator (Weyl) algebra is the enveloping algebra of the relations

$$
\left[y_{\alpha}, y_{\beta}\right]=-2 i \epsilon_{\alpha \beta} .
$$

It can be extended by adding an outer element $K$ anticommuting with $y_{\alpha}$ which is called Klein operator

$$
\left\{y_{\alpha}, K\right\}=0, \quad K K=1 .
$$

With this new element there exists a one-parametric deformation of the algebra

$$
\left[y_{\alpha}, y_{\beta}\right]=-2 i \epsilon_{\alpha \beta}(1+\nu K),
$$

where $\nu$ is an arbitrary constant (central element). Jacobi relations

$$
\left[\left[y_{\alpha}, y_{\beta}\right], y_{\gamma}\right]+\text { cycle }=0
$$

automatically hold for (3.18) provided that indices $\alpha, \beta, \gamma$ range two values. As was stressed already in (2.26) the remarkable property of (3.18) is that for any $\nu$ the bilinears of $y$ generate $s p(2)$

$$
M_{\alpha \beta}=\left\{y_{\alpha}, y_{\beta}\right\}, \quad\left[M_{\alpha \alpha}, M_{\beta \beta}\right] \sim \epsilon_{\alpha \beta} M_{\alpha \beta} .
$$

Adding another copy of deformed oscillator $\bar{y}_{\dot{\alpha}}$ in the dotted sector one extends this $s p(2)$ to the four dimensional Lorentz algebra $s p(2 \mid \mathbb{C})$. Eqs. (3.7)-(3.11) provide a generalization of $(3.18)$ that still respects the $s p(2 \mid \mathbb{C})$ symmetry. The simplest such an extension arises if one sets $B=$ const and $b=0$ in which case the defining relations become

$$
\begin{aligned}
{\left[s_{\alpha}, s_{\beta}\right] } & =-2 i \epsilon_{\alpha \beta}(1+\nu K), & {\left[\bar{s}_{\dot{\alpha}}, \bar{s}_{\dot{\beta}}\right] } & =-2 i \epsilon_{\dot{\alpha} \dot{\beta}}(1+\nu \bar{K}), \\
\left\{s_{\alpha}, K\right\} & =0, & \left\{\bar{s}_{\dot{\alpha}}, \bar{K}\right\} & =0, \\
\left\{t_{\alpha}, K\right\} & =0, & \left\{\bar{t}_{\dot{\alpha}}, \bar{K}\right\} & =0, \\
{\left[s_{\alpha}, \bar{s}_{\dot{\alpha}}\right] } & =0, & {\left[t_{\alpha}, s^{\alpha}\right]+\left[\bar{t}_{\dot{\alpha}}, \bar{s}^{\dot{\alpha}}\right] } & =2 i g K \bar{K} .
\end{aligned}
$$

Since the part of the $s p(2) \oplus s p(2)$ Lorentz transformations in (3.13) and (3.14) associated with $\phi, \bar{\phi}, \psi$ and $\bar{\psi}$ is generated by the gauge symmetries of the higher differential form fields, it can be argued that the Lorentz $s p(2) \oplus s p(2)$ symmetry is still generated by $M_{\alpha \beta}$ (3.20) on the factor algebra of the universal enveloping algebra of (3.21)-(3.24) over the ideal generated by the higher-spin gauge transformations. 


\subsection{Lorentz covariant equations}

To find proper set of fields that brings (3.1), (3.2) into a manifestly Lorentz covariant form we again use the fact that such a field redefinition containing Lorentz connection $\omega_{\alpha \beta}, \bar{\omega}_{\dot{\alpha} \dot{\beta}}$ leaves twistor fields (space-time 0-forms) unaffected. Therefore the covariantized version for space-time evolution of these fields is reproduced by replacing d with $D^{L}$ in the original equations (3.1), (3.2). These in turn impose further restrictions on the other equations via integrability condition (2.48) still not fixing the latter entirely. Skipping technical details let us give the final result for the manifestly Lorentz covariant HS equations

$$
\begin{aligned}
D^{L} \mathcal{W}+\mathcal{W} * \mathcal{W} & +R^{A B}\left(L_{A B}-\frac{i}{4} \partial_{A} \mathcal{W} * \partial_{B} \mathcal{W}\right)=i \theta^{A} \theta_{A}+i \eta \mathcal{B} * \gamma+i \bar{\eta} \mathcal{B} * \bar{\gamma}+i g \gamma \bar{\gamma} \\
& +\mathcal{L}-\frac{\eta}{4} R^{\alpha \beta} \partial_{\alpha} \mathcal{B} * \partial_{\beta} \gamma-\frac{\bar{\eta}}{4} \bar{R}^{\dot{\alpha} \dot{\beta}} \partial_{\dot{\alpha}} \mathcal{B} * \partial_{\dot{\beta}} \bar{\gamma}+\frac{i \eta}{32} R^{\alpha \alpha} R^{\beta \beta} \partial_{\alpha} \partial_{\beta} \mathcal{B} * \partial_{\alpha} \partial_{\beta} \gamma \\
& +\frac{i \bar{\eta}}{32} \bar{R}^{\dot{\alpha} \dot{\alpha}} \bar{R}^{\dot{\beta} \dot{\beta}} \partial_{\dot{\alpha}} \partial_{\dot{\beta}} \mathcal{B} * \partial_{\dot{\alpha}} \partial_{\dot{\beta}} \bar{\gamma} \\
D^{L} \mathcal{B} & +[\mathcal{W}, \mathcal{B}]-\frac{i}{4} R^{A B}\left\{\partial_{A} \mathcal{B}, \partial_{B} \mathcal{W}\right\}_{*}=0
\end{aligned}
$$

Equations (3.25), (3.26) reproduce (3.1), (3.2) at $\omega_{A B}=0$ in which case Lorentz connection is contained in $\mathcal{W}$, being equivalent to the latter up to a field redefinition at $\omega_{A B} \neq 0$. This field redefinition can be found in the form of Abelian infinitesimal Stueckelberg transformation

$$
\begin{aligned}
\delta_{\xi} \omega_{A B}= & \xi_{A B}, \quad \delta_{\xi} \mathcal{B}=\frac{i}{4} \xi^{A B}\left\{\partial_{A} \mathcal{B}, \partial_{B} \mathcal{W}\right\}_{*} \\
\delta_{\xi} \mathcal{W}= & -\xi^{\alpha \beta}\left(L_{\alpha \beta}-\frac{i}{4} \partial_{\alpha} \mathcal{W} * \partial_{\beta} \mathcal{W}\right)-\frac{\eta}{4} \xi^{\alpha \beta} \partial_{\alpha} \mathcal{B} * \partial_{\beta} \gamma \\
& +\frac{i \eta}{32}\left(\xi^{\alpha \alpha} R^{\beta \beta}+\xi^{\beta \beta} R^{\alpha \alpha}\right) \partial_{\alpha} \partial_{\beta} \mathcal{B} * \partial_{\alpha} \partial_{\beta} \gamma+\text { c.c. }
\end{aligned}
$$

To check consistency of (3.25), (3.26) is a bit tiresome and needs to use Fierz identities.

Let us briefly sketch the consistency check. Acting with $D^{L}$ on eqs. (3.25), (3.26) and using (2.48) one arrives at algebraic consequences graded by the degree of the Lorentz curvature $R_{A B}$. One should bear in mind that among different bilinears in fields entering these equations only those actually contribute which do not exceed the differential form degree of $D^{L} \mathcal{W}$ in (3.25) and of $D^{L} \mathcal{B}$ in (3.26). The rest should be discarded. Hence, one is left with $O\left(R^{0}\right), O(R)$ and $O(R \wedge R)$ consistency constraints to be checked independently.

$O\left(R^{0}\right)$ constraints are automatically satisfied since they correspond to the original system (3.1), (3.2) which is consistent. First nontrivial contribution coming from the $O(R)$-terms gives

$$
\left\{\mathcal{B} * \partial_{\alpha} \gamma, \partial_{\alpha} \mathcal{W}\right\}+\left[\partial_{\alpha} \mathcal{B} * \partial_{\alpha} \gamma, \mathcal{W}\right]-\partial_{\alpha}[\mathcal{W}, \mathcal{B}] * \partial_{\alpha} \gamma=0
$$

(similarly for the antiholomorphic part) which is equivalent to

$$
-\left(\mathcal{B} * \partial_{\alpha} \gamma * \partial_{\alpha} \mathcal{W}+\partial_{\alpha} \mathcal{B} * \partial_{\alpha} \gamma * \mathcal{W}\right)=\left(\partial_{\alpha} \mathcal{B} * \mathcal{W}+\mathcal{B} * \partial_{\alpha} \mathcal{W}\right) * \partial_{\alpha} \gamma
$$


Looking at (3.30) we observe that the $\theta^{2}$-components of $\mathcal{W}$ and $\mathcal{B}$ do not contribute because of $\theta^{3} \equiv 0$ and symmetrization $\theta_{\alpha} \theta_{\alpha} \equiv 0$. That $\theta_{\alpha} \theta_{\alpha} \equiv 0$ also implies that the terms with both fields $\mathcal{W}$ and $\mathcal{B}$ being linear in $\theta$ do not contribute. Therefore, only $\mathcal{W}$ linear in $\theta$ components in the first term and $\theta$ - independent in the second survive on the l.h.s. of (3.30). Using $\partial_{\alpha} \gamma * \mathcal{W}=\mathcal{W}(\theta,-\bar{\theta},-d x) * \partial_{\alpha} \gamma=-\mathcal{W} * \partial_{\alpha} \gamma$ for $\theta$-independent components and $\partial_{\alpha} \gamma * \partial_{\alpha} \mathcal{W}=-\partial_{\alpha} \mathcal{W}(\theta,-\bar{\theta},-d x) * \partial_{\alpha} \gamma=-\partial_{\alpha} \mathcal{W} * \partial_{\alpha} \gamma$ for those linear in $\theta$ we find that (3.30) holds true.

For (3.26) the $O(R)$-consistency implies

$$
\left[\mathcal{B}, \partial_{\alpha} \mathcal{B} * \partial_{\alpha} \gamma\right]=\left[\mathcal{B} * \partial_{\alpha} \gamma, \partial_{\alpha} \mathcal{B}\right]
$$

which is also true by similar arguments.

Analyzing consistency of (3.25), (3.26) up to the $R^{2}$ level one has to remember that only differential forms of degree $d x^{4} \theta^{A}$ can show up in $\left(D^{L}\right)^{2} \mathcal{W}+\ldots$ and $d x^{4}$ in $\left(D^{L}\right)^{2} \mathcal{B}+\ldots$ Indeed, the degree of $\mathcal{W}$ is $(p, 3-p)$, where $p=0, \ldots, 3$ is attributed to $d x^{p}$. Therefore, $\#\left(D^{L}\right)^{2} \mathcal{W}=(p+2,3-p)$. Now, the top form $R \wedge R$ implies that $p=2$ and the only terms that should be analyzed within consistency constraints are $d x^{4} \theta^{A}$. The same argument applies to $\left(D^{L}\right)^{2} \mathcal{B}+\ldots$ equation. In practice this has the result that only the twistor sector contribution to the consistency relation has to be checked. This turns out to be fulfilled for (3.25), (3.26). This analysis implies in particular that had the system (3.25), (3.26) contained higher differential forms it would not be Lorentz covariant unless a proper deformation in the sector of higher differential forms is taken into account.

Another interesting point is that as advertised the Lorentz symmetry justifies the choice of the central element $i g \gamma * \bar{\gamma}$ in (3.1). To see that let us denote it by $c$. Then the consistency of (3.25) implies in particular

$$
R^{A B}\left\{\partial_{A} c, \partial_{B} \mathcal{W}\right\}_{*}=0
$$

which is true for $c=i g \gamma * \bar{\gamma}$ but not for other $\theta^{4}$-central elements. For instance, for $c=\theta^{\alpha} \theta_{\alpha} \bar{\theta}^{\dot{\alpha}} \bar{\theta}_{\dot{\alpha}},(3.32)$ is not true any longer. Therefore, (3.25), (3.26) are consistent only for the specific central element $g \gamma * \bar{\gamma}$ that governs the invariant functional density $\mathcal{L}$.

In our analysis of the extended HS equations (3.1), (3.2) we have chosen their interaction phase ambiguity to be a field-independent complex number $\eta$. An extension of the proposed Lorentz covariantization scheme to an arbitrary $F_{*}(\mathcal{B})$ is simple, resulting in minor modification of (3.25), (3.26). Namely, to introduce field-dependent phase in a Lorentz covariant way one simply replaces $\eta \mathcal{B}$ with $F_{*}(\mathcal{B}), \bar{\eta} \mathcal{B}$ with $\bar{F}_{*}(\mathcal{B})$ and $g$ with $G_{*}(\mathcal{B})$ everywhere on the r.h.s. of (3.25) while leaving (3.26) intact. Thus modified equations turn out to remain consistent.

\section{Covariant perturbation theory}

Lorentz covariance of the proposed scheme is manifest provided it is not explicitly broken by the homotopy calculations in the perturbative analysis. This is true for the vast class of Lorentz-covariant homotopies proposed recently in [48] where a subclass of homotopies 
leading to the local vertices was identified. That these homotopies properly reproduce the results of $[49,50]$ is shown in [51]. In this paper we illustrate the usefulness of the proposed formulation using the conventional homotopy allowing us to apply the approach developed recently in [43].

Equations (3.1), (3.2) naturally extend HS equations (2.1), (2.2) in a sense that all new higher-forms come hand in hand with the structure of the original equations. A new ingredient is the central element $i g \gamma * \bar{\gamma}$ giving rise to the nontrivial invariant functional in perturbation theory. It also delivers nontrivial vacuum value for $\mathcal{W}_{0}$ field which turns out to be no longer polynomial in oscillators. However what really complicates the perturbation theory is that the number of elementary homotopy calculations within HS equations grows combinatorially with the degree of differential forms and that the naive result turns out to be involved due to the terms containing background Lorentz connection $\omega_{A B}$.

As shown in [43] one can dodge the first problem by finding explicit expressions for operators originated from repeated homotopy integration. The obtained formulas automatically account combinatorial contributions of terms coming from different differential forms. As for the second problem, the reason why noncovariant terms do show up is that the Lorentz covariance of (3.1), (3.2) is not manifest. These can be eliminated using the field redefinition obtained in (3.27), (3.28). However a much simpler scheme developed in this section is to reconsider the perturbation theory of [43] within the new Lorentz-covariant setup of (3.25), (3.26).

We start with the proper vacuum solution to (3.25), (3.26) corresponding to $A d S_{4}$ space-time,

$$
\mathcal{W}_{0}=\frac{i}{2} e^{\alpha \dot{\alpha}} y_{\alpha} \bar{y}_{\dot{\alpha}}+\theta^{A} Z_{A}+\mathcal{W}_{0}^{3}, \quad R_{0}^{\alpha \beta}=-e_{\dot{\beta}}^{\alpha} \wedge e^{\beta \dot{\beta}}, \quad \mathcal{B}_{0}=0,
$$

where $e^{\alpha \dot{\alpha}}$ is the $A d S_{4}$ vierbein field and $\mathcal{W}_{0}^{3}$ is the vacuum part of the 3 -form which for now is not important and will be specified later on. Using (4.1) as a background in perturbation theory we note that both (3.25) and (3.26) lead to similar equations

$$
D^{L} f+\left[S_{0}, f\right]_{*}+\left[W_{0}, f\right]_{*}-\frac{i}{4} R^{A B}\left\{\partial_{A} f, \partial_{B} S_{0}\right\}_{*}=J
$$

Here, $S_{0}=\theta^{A} Z_{A}, W_{0}=\frac{i}{2} e^{\alpha \dot{\alpha}} y_{\alpha} \bar{y}_{\dot{\alpha}}$ and $f$ is either $\mathcal{W}$ or $\mathcal{B}$, while $J$ denotes the leftover terms appearing in (3.25) or (3.26). The dependence on extra Klein operators in $f$ leads to different realization of the commutator $\left[W_{0}, f\right]$ driving to the adjoint or twisted-adjoint representations. It is important to note that all of the remaining terms on the l.h.s. of (4.2) act evenly as differential operators in these two possible cases. Consider the two cases in more detail.

\subsection{Adjoint case}

In this case $f$ is either independent of $k$ and $\bar{k}$ or bilinear $f \rightarrow f k \bar{k}$. We separate the dependence on kleinians so as to have $f$ Klein independent. One then has

$$
\mathcal{D} f-2 i \theta^{A} \frac{\partial}{\partial Z^{A}} f=J
$$


where

$$
\begin{aligned}
\mathcal{D}= & \mathrm{d}+\omega_{L}^{A B}\left(Y_{A} \frac{\partial}{\partial Y^{B}}+Z_{A} \frac{\partial}{\partial Z^{B}}+\theta_{A} \frac{\partial}{\partial \theta^{B}}\right)-\frac{i}{2} R^{A B}\left(Z_{A} \frac{\partial}{\partial \theta^{B}}+i \frac{\partial}{\partial Y^{A}} \frac{\partial}{\partial \theta^{B}}\right) \\
& -e^{A B}\left(Y_{A} \frac{\partial}{\partial Y^{B}}-i \frac{\partial}{\partial Z^{A}} \frac{\partial}{\partial Y^{B}}\right) .
\end{aligned}
$$

$f$ can be found from (4.3) up to a purely gauge part as

$$
f=\Delta^{-1} J+g, \quad \mathcal{D} g=\left.(J-\mathcal{D} f)\right|_{Z=\theta=0}=: \mathcal{H} J
$$

where $g$ is $Z$ - and $\theta$-independent $\mathrm{d}_{Z^{-}}$-cohomology part of $\mathrm{d}_{Z}:=\theta^{A} \frac{\partial}{\partial Z^{A}}$ (see [43] for more detail) and formal inversion of operator $\Delta=\mathcal{D}-2 i \mathrm{~d}_{Z}$ amounts to

$$
\Delta^{-1} J=-\frac{1}{2 i} \sum_{n=0}^{\infty}\left(\frac{\mathrm{d}_{Z}^{*} \mathcal{D}}{2 i}\right)^{n} \mathrm{~d}_{Z}^{*} J, \quad \mathrm{~d}_{Z}^{*} J=Z^{A} \frac{\partial}{\partial \theta^{A}} \int_{0}^{1} \frac{d t}{t} J(t Z, Y ; t \theta) .
$$

Since $\left(\mathrm{d}_{Z}^{*}\right)^{2}=0$, everything in $\mathcal{D}$ that commutes with $\mathrm{d}_{Z}^{*}$ brings no contribution to $\Delta^{-1}$. The last term in the first line of (4.4) that contains curvature $R^{A B}$ while not commuting with $\mathrm{d}_{Z}^{*}$ still adds no contribution to $\Delta^{-1}$. This follows from $\mathrm{d}_{Z}^{*} R^{A B}\left(Z_{A} \frac{\partial}{\partial \theta^{B}}+i \frac{\partial}{\partial Y^{A}} \frac{\partial}{\partial \theta^{B}}\right) \mathrm{d}_{Z}^{*} J \equiv 0$. Hence, the only term that matters in $\mathcal{D}$ is

$$
\mathcal{D} \sim i e^{A B} \frac{\partial}{\partial Z^{A}} \frac{\partial}{\partial Y^{B}} .
$$

This way one finally gets

$$
\begin{aligned}
\Delta_{a d}^{-1} J & =-\frac{1}{2 i} Z^{A} \frac{\partial}{\partial \theta^{A}} \int_{0}^{1} \frac{d t}{t} e^{\frac{1-t}{2 t} e^{B C} \frac{\partial^{2}}{\partial Y^{B} \partial \theta^{C}} J(t Z, Y ; t \theta)} \\
& =-\frac{1}{2 i} Z^{A} \frac{\partial}{\partial \theta^{A}} \int_{0}^{1} \frac{d t}{t} J\left(t Z, Y_{A}+\frac{1-t}{2 t} e_{A}^{B} \frac{\partial}{\partial \theta^{B}} ; t \theta\right) .
\end{aligned}
$$

Substituting (4.8) into the r.h.s. of the second equation (4.5) one finds the projection to cohomology operator

$$
\mathcal{H}_{a d} J=\left.J\left(0, Y_{A}+\frac{1}{2} e_{A}{ }^{B} \frac{\partial}{\partial \theta^{B}} ; \theta\right)\right|_{\theta=0} .
$$

Remarkably the $R$-curvature term does not enter perturbative operators (4.8), (4.9) at all as one reproduces formulas from [43] with the Lorentz connection set to zero.

A systematic way of obtaining explicit form of homotopy operators which is most useful in twisted-adjoint case was proposed in [43] as we briefly recall. Consider a general equation of the form

$$
\Delta f:=\mathrm{d} f+\mathcal{D} f=J,
$$

where

$$
\Delta^{2}=0, \quad \mathrm{~d}^{2}=0 .
$$

The consistency of (4.10) requires

$$
\mathrm{d} J+\mathcal{D} J=0 .
$$


Then, if a resolution of identity for $\mathrm{d}$

$$
\left\{\mathrm{d}, \mathrm{d}^{*}\right\}+h=I d
$$

is known and

$$
\left\{\mathrm{d}^{*}, \mathcal{D}\right\}=0
$$

holds true, a general solution to (4.10) can be written. To this end one applies (4.13) to $J$ in (4.10) and, making use of (4.12) and (4.14), rewrites it as

$$
\mathrm{d}\left(f-\mathrm{d}^{*} J\right)+\mathcal{D}\left(f-\mathrm{d}^{*} J\right)=h J .
$$

A general solution to (4.15) is

$$
f=\mathrm{d}^{*} J+g+\Delta \epsilon+\chi
$$

where an arbitrary function $\epsilon$ and general cohomology $\chi \in H(\Delta)$ constitute a general solution to the homogeneous equation, while $g$ solves

$$
h \mathcal{D} g=h J .
$$

This can be used to build a resolution of identity for the operator from (4.3). We have $\Delta_{a d}=\mathcal{D}_{a d}-2 i \mathrm{~d}_{Z}$, where $\mathrm{d}_{Z}=\theta^{A} \frac{\partial}{\partial Z^{A}}$. For $\mathrm{d}_{Z}$ one has

$$
\begin{aligned}
\mathrm{d}^{*} f(Z ; \theta) & =Z^{A} \frac{\partial}{\partial \theta^{A}} \int_{0}^{1} \frac{d t}{t} f(t Z ; t \theta), \\
h f(Z ; \theta) & =f(0 ; 0) .
\end{aligned}
$$

The nilpotency of $\Delta$ can be check straightforwardly. However, in this case (4.14) does not hold due to the terms $\frac{1}{2} R^{A B} \frac{\partial^{2}}{\partial Y^{A} \partial \theta^{B}}$ and $i e^{A B} \frac{\partial^{2}}{\partial Z^{A} \partial Y^{B}}$ in (4.4), that do not anticommute with $\mathrm{d}^{*}$.

To bypass this obstacle we perform a similarity transformation on the space of functions in question

$$
f(Z ; Y ; \theta) \longrightarrow \widetilde{f}(Z ; Y ; \theta)=\exp \left\{-e^{A B} \frac{\partial^{2}}{\partial Y^{A} \partial \theta^{B}}\right\} f(Z ; Y ; \theta) .
$$

In terms of wavy functions (4.3) turns to

$$
D^{L} \widetilde{f}-e^{A B} Y_{A} \frac{\partial}{\partial Y^{B}} \widetilde{f}-2 i \mathrm{~d}_{Z} \widetilde{f}+R^{A B} Z_{A} \frac{\partial}{\partial \theta^{B}} \widetilde{f}=\widetilde{J} .
$$

Now though containing $R$-term, the operator $\mathcal{D}$ in (4.21) results in (4.14) being satisfied, and the general solution is provided by (4.16). Performing inverse transformation, one finds

$$
f=\exp \left\{e^{A B} \frac{\partial^{2}}{\partial Y^{A} \partial \theta^{B}}\right\} \mathrm{d}^{*} \exp \left\{-e^{A B} \frac{\partial^{2}}{\partial Y^{A} \partial \theta^{B}}\right\} J+g+\Delta_{a d} \epsilon,
$$

where $g(Y)$ solves

$$
D^{L} g+e^{A B} Y_{A} \frac{\partial}{\partial Y^{B}} g=h\left(\exp \left\{-e^{A B} \frac{\partial^{2}}{\partial Y^{A} \partial \theta^{B}}\right\} J\right) .
$$


Substituting (4.18) and simplifying, one recovers (4.8) and (4.9). Applying resolution of identity (4.13) to some $\tilde{f}$ one finds that in terms of corresponding $f$ it amounts to

$$
\left\{\Delta_{a d}, \Delta_{a d}^{-1}\right\}+\mathcal{H}_{a d}=I d
$$

Formula (4.8) enables us to complete vacuum solution (4.1). Indeed at the lowest order we have

$$
\Delta_{a d} \mathcal{W}_{0}^{3}=i g \gamma * \bar{\gamma}
$$

and since $\mathcal{H}_{a d}(\gamma * \bar{\gamma})=0$, from (4.8) one finds

$$
\begin{aligned}
\mathcal{W}_{0}^{3} & =-2 g Z^{A} \frac{\partial}{\partial \theta^{A}} \int_{0}^{1} d t t^{3} e^{i t Z_{A} Y^{A}+i(1-t) \frac{1}{2} Z_{A} e^{A B} \frac{\partial}{\partial \theta^{B}}} \delta^{4}(\theta) \\
& =-2 g Z^{A} \int_{0}^{1} d t t^{3} e^{i t Z_{A} Y^{A}} \delta_{A}^{\prime}\left(\theta_{B}+i(1-t) \frac{1}{2} e_{B}{ }^{C} Z_{C}\right) k \bar{k}
\end{aligned}
$$

Formally, this expression for the vacuum 3-form coincides with that of [43] with Lorentz connection $\omega_{A B}^{L}$ set to zero. But these two expressions represent partial solutions for two different systems of equations, related by Stueckelberg transformation (3.27), (3.28). Thus, in general, one could expect that, for instance, the curvature $R^{A B}$ will appear in (4.26), as it enters (3.28). However, this does not happen because, as we have shown, $R$-dependent terms do not contribute to homotopy operators. So terms with Lorentz curvature may appear in the vacuum 3 -form only via $\Delta_{a d}$-exact additions to (4.26). It can be shown however that (4.26) is literally reproduced from that of [43] by Stueckelberg transformation (3.28).

Analogously in the twisted-adjoint case the perturbative operators repeat those from [43] with $\omega_{A B}^{L}=0$ leading to a significant simplification of the operator form as we now show.

\subsection{Twisted-adjoint case}

In this case $f \sim f_{1} k+f_{2} \bar{k}$. Separating again the dependence on $k$ and $\bar{k}$ and abusing notation renaming $f_{1,2} \rightarrow f$ one finds that commutator $\left[W_{0}, f\right]$ turns into anticommutator

$$
\frac{i}{2} e^{\alpha \dot{\alpha}}\left\{y_{\alpha} \bar{y}_{\dot{\alpha}}, f\right\}=\frac{i}{2} e^{A B}\left(Y_{A} Y_{B}-\frac{\partial^{2}}{\partial Y^{A} \partial Y^{B}}-2 i Y_{A} \frac{\partial}{\partial Z^{B}}-\frac{\partial^{2}}{\partial Z^{A} \partial Z^{B}}\right)
$$

so that

$$
\begin{aligned}
\mathcal{D}_{t w}= & \mathrm{d}+\omega_{L}^{A B}\left(Y_{A} \frac{\partial}{\partial Y^{B}}+Z_{A} \frac{\partial}{\partial Z^{B}}+\theta_{A} \frac{\partial}{\partial \theta^{B}}\right)-\frac{i}{2} R^{A B}\left(Z_{A} \frac{\partial}{\partial \theta^{B}}+i \frac{\partial}{\partial Y^{A}} \frac{\partial}{\partial \theta^{B}}\right) \\
& +\frac{i}{2} e^{A B}\left(Y_{A} Y_{B}-\frac{\partial^{2}}{\partial Y^{A} \partial Y^{B}}-2 i Y_{A} \frac{\partial}{\partial Z^{B}}-\frac{\partial^{2}}{\partial Z^{A} \partial Z^{B}}\right)
\end{aligned}
$$

Once again, the contribution that does not anticommute with $\mathrm{d}_{Z}^{*}$ is

$$
\mathcal{D}_{t w} \sim e^{A B}\left(Y_{A} \frac{\partial}{\partial Z^{B}}-\frac{i}{2} \frac{\partial^{2}}{\partial Z^{A} \partial Z^{B}}\right)+\frac{1}{2} R^{A B} \frac{\partial^{2}}{\partial \theta^{A} \partial Y^{B}}
$$


and can be compensated by the following transformation

$$
f(Z ; Y ; \theta) \longrightarrow \widetilde{f}(Z ; Y ; \theta)=\exp \left\{i e^{A B} Y_{A} \frac{\partial}{\partial \theta^{B}}+\frac{1}{2} e^{A B} \frac{\partial^{2}}{\partial Z^{A} \partial \theta^{B}}\right\} f(Z ; Y ; \theta) .
$$

For wavy functions one has

$$
D^{L} \widetilde{f}+\frac{i}{2} e^{A B}\left(Y_{A} Y_{B}-\frac{\partial^{2}}{\partial Y^{A} \partial Y^{B}}\right) \tilde{f}-2 i \mathrm{~d}_{Z} \widetilde{f}-\frac{i}{2} R^{A B} Z_{A} \frac{\partial}{\partial \theta^{B}} \widetilde{f}=\widetilde{J},
$$

that satisfies (4.14) and thus is solved by (4.16).

Inversion of (4.30) leads to the following operators (4.5)

$$
\begin{aligned}
& \Delta_{t w}^{-1} J=-\frac{1}{2 i} Z^{A} \frac{\partial}{\partial \theta^{A}} \int_{0}^{1} \frac{d t}{t} e^{-i \frac{1-t}{2 t} e^{A B} Y_{A} \frac{\partial}{\partial \theta^{B}}-\frac{1-t^{2}}{4 t^{2}} e^{A B} \frac{\partial^{2}}{\partial Z^{A} \partial \theta^{B}} J(t Z, Y ; t \theta),} \\
& \mathcal{H}_{t w} J=e^{-\frac{i}{2} e^{A B} Y_{A} \frac{\partial}{\partial \theta^{B}}-\left.\frac{1}{4} e^{A B} \frac{\partial^{2}}{\partial Z^{A} \partial \theta^{B}} J(Z, Y ; \theta)\right|_{Z=\theta=0},}
\end{aligned}
$$

which provide a resolution of identity

$$
\left\{\Delta_{t w}, \Delta_{t w}^{-1}\right\}+\mathcal{H}_{t w}=I d,
$$

determining the perturvative expansion in the twisted-adjoint sector.

An important comment is as follows. The elaborated perturbation theory is based on the conventional resolution $d_{Z}^{*}$, (4.6) which is known to lead to non-localities in HS interactions at second order on the equations of motion first observed in [34] and later on shown more systematically in [50,52]. This raises a serious question on the class of admissible functions, which is not a subject of current investigation. Nevertheless at least from technical stand point the conventional resolution can be a useful tool in a search for the aforementioned class of functions. For example in [49] and [53] it was used in derivation of local HS cubic interactions.

\section{Conclusion}

Let us summarize the main findings of this work. It is shown that HS extended equations of [30] having room for gauge invariant functionals admit local Lorentz symmetry. This symmetry gets manifest upon field redefinition leading to the explicitly Lorentz covariant form of HS equations. In obtaining Lorentz frame we notice that its consistency condition is more severe that the standard unfolded one ruling out certain HS systems that otherwise are formally consistent within the unfolded frame. This fact provides a double check of the central element 4-form in (3.1) chosen in [28] when constructing generalized HS equations containing invariant functional. Unlike the 2-form central element of the original HS equations (2.1) which can be tested by its consistency with free HS equations, the central 4-form can not be checked by the linearization as it belongs to a higher-form sector responsible for the invariant functional rather than dynamical equations.

The existence of Lorentz frame for system (3.1), (3.2) though mandatory due to equivalence principle is not quite trivial since it imposes an overdetermined set of constraints 
being all fulfilled. Particulary, while higher-form extension of the equations of [2] admits certain freedom in a top-form central term from mere consistency, the only one of those is compatible with the obtained Lorentz covariant equations. A key element of the construction intrinsically responsible for Lorentz symmetry is the twistor sector (algebraic constraints for space-time 0-form fields) of the HS extended equations. The twistor sector of the original HS equations is known to generate Lorentz symmetry through the deformed oscillators. So does the extended twistor sector (3.7), (3.11). Supporting Lorentz symmetry it represents a higher-dimensional generalization of the deformed oscillators underling nonlinear HS equations which deserves special attention in the context of Calogero-like models and perhaps higher-dimensional HS equations.

A byproduct of the obtained Lorentz frame is a significant simplification of the HS perturbation theory despite seemingly involved form of the covariant equations. We have reconsidered the analysis of [43] and found the covariant version of the perturbative series operators to gain a simpler form within the new approach. This significantly simplifies calculation of the invariant functional density 4 -form at least to lowest orders.

\section{Acknowledgments}

The research was supported by the Russian Science Foundation grant 14-42-00047 in association with Lebedev Physical Institute. The work of N.G.M. was partially supported by the Foundation for Theoretical Physics Development "Basis".

Open Access. This article is distributed under the terms of the Creative Commons Attribution License (CC-BY 4.0), which permits any use, distribution and reproduction in any medium, provided the original author(s) and source are credited.

\section{References}

[1] M.A. Vasiliev, Consistent equation for interacting gauge fields of all spins in (3+1)-dimensions, Phys. Lett. B 243 (1990) 378 [INSPIRE].

[2] M.A. Vasiliev, More on equations of motion for interacting massless fields of all spins in (3+1)-dimensions, Phys. Lett. B 285 (1992) 225 [INSPIRE].

[3] M.A. Vasiliev, Equations of motion of interacting massless fields of all spins as a free differential algebra, Phys. Lett. B 209 (1988) 491 [INSPIRE].

[4] M.A. Vasiliev, Consistent equations for interacting massless fields of all spins in the first order in curvatures, Annals Phys. 190 (1989) 59 [INSPIRE].

[5] M.A. Vasiliev, Higher spin gauge theories: star product and AdS space, hep-th/9910096 [INSPIRE].

[6] N. Boulanger, C. Iazeolla and P. Sundell, Unfolding mixed-symmetry fields in AdS and the BMV conjecture: I. General formalism, JHEP 07 (2009) 013 [arXiv:0812.3615] [INSPIRE].

[7] C. Fronsdal, Massless fields with integer spin, Phys. Rev. D 18 (1978) 3624 [INSPIRE].

[8] F.A. Berends, G.J.H. Burgers and H. van Dam, On the theoretical problems in constructing interactions involving higher spin massless particles, Nucl. Phys. B 260 (1985) 295 [INSPIRE]. 
[9] R. Manvelyan, K. Mkrtchyan and W. Rühl, General trilinear interaction for arbitrary even higher spin gauge fields, Nucl. Phys. B 836 (2010) 204 [arXiv: 1003.2877] [InSPIRE].

[10] R. Manvelyan, K. Mkrtchyan and W. Ruehl, A Generating function for the cubic interactions of higher spin fields, Phys. Lett. B 696 (2011) 410 [arXiv:1009.1054] [INSPIRE].

[11] E. Joung, L. Lopez and M. Taronna, On the cubic interactions of massive and partially-massless higher spins in (A)dS, JHEP 07 (2012) 041 [arXiv: 1203.6578] [INSPIRE].

[12] D. Francia, G.L. Monaco and K. Mkrtchyan, Cubic interactions of Maxwell-like higher spins, JHEP 04 (2017) 068 [arXiv:1611.00292] [INSPIRE].

[13] C. Sleight and M. Taronna, Higher spin interactions from conformal field theory: the complete cubic couplings, Phys. Rev. Lett. 116 (2016) 181602 [arXiv:1603.00022] [INSPIRE].

[14] A.K.H. Bengtsson, I. Bengtsson and L. Brink, Cubic interaction terms for arbitrary spin, Nucl. Phys. B 227 (1983) 31 [inSPIRE].

[15] A.K.H. Bengtsson, I. Bengtsson and N. Linden, Interacting Higher Spin Gauge Fields on the Light Front, Class. Quant. Grav. 4 (1987) 1333 [InSPIRE].

[16] R.R. Metsaev, Generating function for cubic interaction vertices of higher spin fields in any dimension, Mod. Phys. Lett. A 8 (1993) 2413 [INSPIRE].

[17] R.R. Metsaev, Cubic interaction vertices of massive and massless higher spin fields, Nucl. Phys. B 759 (2006) 147 [hep-th/0512342] [INSPIRE].

[18] R.R. Metsaev, Cubic interaction vertices for fermionic and bosonic arbitrary spin fields, Nucl. Phys. B 859 (2012) 13 [arXiv:0712.3526] [INSPIRE].

[19] D. Ponomarev, Off-shell spinor-helicity amplitudes from light-cone deformation procedure, JHEP 12 (2016) 117 [arXiv:1611.00361] [INSPIRE].

[20] A.K.H. Bengtsson, Investigations into light-front quartic interactions for massless fields (I): non-constructibility of higher spin quartic amplitudes, JHEP 12 (2016) 134 [arXiv: 1607.06659] [INSPIRE].

[21] M. Taronna, On the non-local obstruction to interacting higher spins in flat space, JHEP 05 (2017) 026 [arXiv: 1701.05772] [INSPIRE].

[22] D. Ponomarev and E.D. Skvortsov, Light-front higher-spin theories in flat space, J. Phys. A 50 (2017) 095401 [arXiv: 1609. 04655] [INSPIRE].

[23] D. Ponomarev, Chiral higher spin theories and self-duality, JHEP 12 (2017) 141 [arXiv:1710.00270] [INSPIRE].

[24] R.R. Metsaev, Poincaré invariant dynamics of massless higher spins: fourth order analysis on mass shell, Mod. Phys. Lett. A 6 (1991) 359 [inSPIRE].

[25] M.A. Vasiliev, 'Gauge' form of description of massless fields with arbitrary spin (in Russian), Yad. Fiz. 32 (1980) 855 [Sov.J.Nucl.Phys. 32 (1980) 439] [INSPIRE].

[26] M.A. Vasiliev, Free massless fields of arbitrary spin in the de Sitter space and initial data for a higher spin superalgebra, Fortsch. Phys. 35 (1987) 741 [Yad.Fiz. 45 (1987) 1784] [InSPIRE].

[27] E.S. Fradkin and M.A. Vasiliev, On the gravitational interaction of massless higher spin fields, Phys. Lett. B 189 (1987) 89 [INSPIRE].

[28] M.A. Vasiliev, Properties of equations of motion of interacting gauge fields of all spins in $(3+1)$-dimensions, Class. Quant. Grav. 8 (1991) 1387 [INSPIRE]. 
[29] E. Sezgin and P. Sundell, Analysis of higher spin field equations in four-dimensions, JHEP 07 (2002) 055 [hep-th/0205132] [INSPIRE].

[30] M.A. Vasiliev, Invariant functionals in higher-spin theory, Nucl. Phys. B 916 (2017) 219 [arXiv: 1504.07289] [INSPIRE].

[31] I.R. Klebanov and A.M. Polyakov, AdS dual of the critical $\mathrm{O}(N)$ vector model, Phys. Lett. B 550 (2002) 213 [hep-th/0210114] [INSPIRE].

[32] E. Sezgin and P. Sundell, Holography in $4 D$ (super) higher spin theories and a test via cubic scalar couplings, JHEP 07 (2005) 044 [hep-th/0305040] [INSPIRE].

[33] R.G. Leigh and A.C. Petkou, Holography of the $N=1$ higher spin theory on AdS $S_{4}$, JHEP 06 (2003) 011 [hep-th/0304217] [INSPIRE].

[34] S. Giombi and X. Yin, Higher spin gauge theory and holography: the three-point functions, JHEP 09 (2010) 115 [arXiv:0912.3462] [INSPIRE].

[35] J. Maldacena and A. Zhiboedov, Constraining conformal field theories with a higher spin symmetry, J. Phys. A 46 (2013) 214011 [arXiv:1112.1016] [INSPIRE].

[36] S. Giombi and X. Yin, The Higher Spin/Vector Model Duality, J. Phys. A 46 (2013) 214003 [arXiv: 1208.4036] [INSPIRE].

[37] R. de Mello Koch, A. Jevicki, K. Jin and J.P. Rodrigues, $A d S_{4} / C F T_{3}$ construction from collective fields, Phys. Rev. D 83 (2011) 025006 [arXiv: 1008.0633] [inSPIRE].

[38] J.-B. Bae, E. Joung and S. Lal, A note on vectorial AdS $S_{5} / C F T_{4}$ duality for spin-j boundary theory, JHEP 12 (2016) 077 [arXiv:1611.00112] [INSPIRE].

[39] A.A. Tseytlin, On partition function and Weyl anomaly of conformal higher spin fields, Nucl. Phys. B 877 (2013) 598 [arXiv:1309.0785] [inSPIRE].

[40] S. Giombi and I.R. Klebanov, One loop tests of higher spin AdS/CFT, JHEP 12 (2013) 068 [arXiv: 1308.2337] [INSPIRE].

[41] S. Giombi, I.R. Klebanov and A.A. Tseytlin, Partition functions and Casimir energies in higher spin $A d S_{d+1} / C F T_{d}$, Phys. Rev. D 90 (2014) 024048 [arXiv: 1402.5396] [INSPIRE].

[42] N. Boulanger and P. Sundell, An action principle for Vasiliev's four-dimensional higher-spin gravity, J. Phys. A 44 (2011) 495402 [arXiv:1102.2219] [INSPIRE].

[43] V.E. Didenko, N.G. Misuna and M.A. Vasiliev, Perturbative analysis in higher-spin theories, JHEP 07 (2016) 146 [arXiv: 1512.04405] [INSPIRE].

[44] C. Iazeolla and P. Sundell, Families of exact solutions to Vasiliev's $4 D$ equations with spherical, cylindrical and biaxial symmetry, JHEP 12 (2011) 084 [arXiv:1107.1217] [INSPIRE].

[45] M.A. Vasiliev, Quantization on sphere and high spin superalgebras, JETP Lett. 50 (1989) 374 [Pisma Zh. Eksp.Teor.Fiz. 50 (1989) 344] [INSPIRE].

[46] M.A. Vasiliev, Higher spin algebras and quantization on the sphere and hyperboloid, Int. J. Mod. Phys. A 6 (1991) 1115 [inSPIRE].

[47] S.F. Prokushkin and M.A. Vasiliev, Higher spin gauge interactions for massive matter fields in 3D AdS space-time, Nucl. Phys. B 545 (1999) 385 [hep-th/9806236] [INSPIRE].

[48] O.A. Gelfond and M.A. Vasiliev, Homotopy operators and locality theorems in higher-spin equations, arXiv:1805.11941 [INSPIRE]. 
[49] M.A. Vasiliev, Current interactions and holography from the 0-form sector of nonlinear higher-spin equations, JHEP 10 (2017) 111 [arXiv:1605.02662] [INSPIRE].

[50] M.A. Vasiliev, On the local frame in nonlinear higher-spin equations, JHEP 01 (2018) 062 [arXiv: 1707.03735] [INSPIRE].

[51] V.E. Didenko, O.A. Gelfond, A.V. Korybut and M.A. Vasiliev, Homotopy properties and lower-order vertices in higher-spin equations, arXiv:1807.00001 [INSPIRE].

[52] N. Boulanger, P. Kessel, E.D. Skvortsov and M. Taronna, Higher spin interactions in four-dimensions: Vasiliev versus Fronsdal, J. Phys. A 49 (2016) 095402 [arXiv: 1508. 04139] [INSPIRE].

[53] O.A. Gelfond and M.A. Vasiliev, Current interactions from the one-form sector of nonlinear higher-spin equations, Nucl. Phys. B 931 (2018) 383 [arXiv:1706.03718] [INSPIRE]. 